\title{
Targeting of BUB1b Gene Expression in Sentinel Lymph Node Biopsies of Invasive Breast Cancer in Iranian Female Patients
}

\author{
Neda Mansouri ${ }^{1}$, Abolfazl Movafagh ${ }^{1 *}$, Arezou Sayad ${ }^{1}$, Atefeh Heidary \\ Pour $^{2}$, Mohammad Taheri', Shahrzad Soleimani', Hamid Reza Mirzaei ${ }^{3}$, \\ Shohreh Alizadeh Shargh ${ }^{4}$, Eznollah Azargashb ${ }^{5}$, Haleh Bazmi ${ }^{6}$, Hossein Allah \\ Moradi $^{7}$, Fatemeh Zandnia ${ }^{8}$, Mehrdad Hashemi ${ }^{9}$, Nilofar Massoudi ${ }^{10}$, SA \\ Mortazavi-Tabatabaei ${ }^{11}$
}

\begin{abstract}
Detection of micrometastasis in sentinel lymph nodes (SLNs) is a very useful tool for appropriate assessment of the clinical stage of disease in breast cancer patients. Early identification of clinically relevant disease could lead to early treatment or staging approaches for breast cancer patient. Micrometastases in SLNs of women with invasive breast cancer are of great significance in this context. In this study we examined SLN biopsies considered to have small numbers of cancerous cells by real time RT-PCR. All of the samples underwent immunohistochemical staining for cytokeratin for confirmation of the presence or absence of micrometastases. BUB1b expression assay of selected patients with and without metastasis showed overexpression in the former, but not in normal breast and lymph node tissue. Our results may be taken into account in the discussion about the merits of routine use of molecular assessment in pathogenetic studies of SLNs.
\end{abstract}

Keywords: Breast cancer - micro-metastasis - sentinel lymph node - real time PCR - Iranian population

Asian Pac J Cancer Prev, 17, Cancer Control in Western Asia Special Issue, 317-321

\section{Introduction}

Breast cancer is a heterogeneous and complex disease with variable clinical outcomes and has high prevalence in the world (Freedman et al., 2015). The prognosis of this depends on clinical parameters such as age, sex and pathological factors such as tumor size and lymph node status and tumor grade and stage. With the increasing knowledge of the molecular pathways involved in breast cancer, development of new and more accurate methods to predict the tumor condition and its response to treatments become possible and the invasive potential of the tumor could be evaluated (Habel et al., 2013). Detection of tumor in the axillary lymph nodes in patients with breast cancer is an important approach in clinical grading and prognosis of breast cancer disease (Patani and Mokbel, 2011). In addition, the existence of tumor in sentinel lymph nodes derived from the assessed tumor tissue demonstrates the extent of lymph node involvement
(Gerber et al., 2011). In this way, risk is lower in breast cancer patients with initial test results nodes negative biopsy than those that their sentinel lymph nodes results were positive regarding the presence of a small number of cancer cells so called micro-metastases (Guillén-Paredes et al., 2011). In addition, breast cancer patients with micro-metastasis negative sentinel lymph nodes do not requires surgery and Axillary lymph nodes dissection and thus do not suffers from the after effects of axillary lymph nodes removal (EBCTCG, 2005). Evaluation of micro-metastases in the sentinel lymph nodes is carried out by the conventional pathology tests such as $\mathrm{H}$ and $\mathrm{E}$ or the more advanced techniques such as Immunohistochemistry (IHC) with accuracy lower than 90\% (Daniele et al., 2009). Furthermore, the Real time PCR technique with accuracy over $95 \%$ has been used to detect the expression of genes involved in tumor invasion in order to evaluates the sentinel lymph nodes micro-metastases (Gillanders et al., 2004). Also, Downregulation of E-cadherin expression

${ }^{1}$ Department of Medical Genetics, School of Medicine, ${ }^{3}$ Department of Radiation Oncology, Cancer Research Center, Shohadae Tajrish Hospital, ${ }^{5}$ Department of Epidemiology, School of Medicine, ${ }^{10}$ Department of Anesthesiology, ${ }^{11}$ Proteomics Research Center, School of Paramedical Sciences, Shahid Beheshti University of Medical Sciences, ${ }^{2}$ Department of Cell and Molecular Biology, Pharmaceutical Sciences Branch, ${ }^{9}$ Department of Molecular Genetics, Islamic Azad University, ${ }^{7}$ Medical research fellow, Tehran, ${ }^{4}$ Department of Medical Laboratory Science, Midwifery-Nursing Institute, Islamic Azad University of Chalous Branch, Chalous, ${ }^{6}$ Department of Biology Laboratory Science, Islamic Azad University of Roudehen Branch, Roudehen, ${ }^{8}$ Department of Biology, Shahre kord University of Islamic Azad University, Shahrekord, Iran. FFor correspondence: movafagh.a@sbmu.ac.ir 
in breast cancer by promoter hypermethylation and its relation with progression and prognosis of tumor in SLN also reported from this center (Shargh et al., 2014). This study was conducted for the first time in an Iranian women suffering from breast cancer with the expression of BUB1 gene with employing by Real-Time PCR technique.

\section{Materials and Methods}

We received breast cancer specimen from 50 women (mean age of $35.2 \pm 70.6$ years) who had undergone surgery at Shohadaye Tajrish-based referral and teaching hospital affiliated to Shahid Beheshti University of Medical Sciences, Tehran Iran from Jun 2013 to December 2014. Also, normal breast tissues $(\mathrm{N}=50)$ was taken from the same patient's breast that undergone partial or total mastectomy. Data for all patients were collected for analysis. All of them diagnosis for breast cancer and without metastasis that tested by IHC staining.

Study and patients were assigned on the basis of national/international breast cancer protocols and approved according to local law and regulations, by the Institutional Review Boards of each participating referral hospital.

\section{Total RNA Isolation and cDNA Synthesis}

Genomic DNA and total RNA from each sample were extracted using a QIAamp DNA mini Kit (Qiagen, Germantown, MD) and an RNeasy mini kit (Qiagen, Germantown, MD), were used according to the manufacturer's instructions. The extracted genomic DNA and total RNA were quantified and confirmed for OD 260/280 values between 1.8 and 2.2 and OD 260/230 values greater than 1 .

Whole cell RNA isolation from sentinel lymph nodes of breast cancer patient were performed using and homogenized reagent, nearly $50 \mu \mathrm{gr}$ of SLN specimen was added to $1 \mathrm{~mL}$ of reagent and homogenized. Then incubation from 5 minutes at room temperature and then added $0.2 \mathrm{~mL}$ of chloroform and homogenized and centrifuged in $12000 \mathrm{rpm}$ for 15 minutes at $4{ }^{\circ} \mathrm{C}$. RNA was precipitated retained and added isopropyl alcohol. Upper aqueous phase was removed. Then centrifuged in 12,000rpm For 10 minutes at $4{ }^{\circ} \mathrm{C}$, further pellet was rinsed twice with $1 \mathrm{~mL}$ of ethanol (75\%). The RNA was re-suspended in DEPS water to a concentration of $0.5 \mu \mathrm{g} /$ $\mu \mathrm{L}$.

\section{Real-time quantitative polymerase chain reaction}

The amount of $0.5 \mu \mathrm{g}$ of oligo DT and $16 \mu \mathrm{L}$ RNase free water added to $5 \mu \mathrm{gr}$ of whole RNA and incubated for 10 minute. RNA extraction was performed using the Qiagen RNeasy Plus Mini Kit (Qiagen, Germantown, MD) according to the manufacturers instructions. Total RNA concentration was measured spectrophotometrically and equal concentrations were added to each well of a 96-well PCR plate. RT-qPCR was performed using the BioRad iTaq reagent in a Bio-Rad CFX96 (Bio-Rad, Hercules, CA). The primers for SYBR Green real-time PCR were designed specific for each checkpoint gene and for ACTB gene ( $\beta$-actin) as internal control. The assays were repeated in their entirety for each measurement. Reverse Transcription is carried out with the Super Script First-Strand Synthesis System for RT-PCR. The following procedure is based on following to the manufacturers instructions, (total RNA $5 \mu \mathrm{g}$, random hexamers (50 ng/

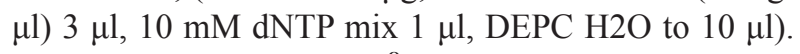
Incubate the samples at $65^{\circ} \mathrm{C}$ for $5 \mathrm{~min}$ and then on ice for at least $1 \mathrm{~min}$. Prepare reaction master mixture. For each reaction (10x RT buffer $2 \mu \mathrm{l}, 25 \mathrm{mM} \mathrm{MgCl} 24 \mu \mathrm{l}$, $0.1 \mathrm{M}$ DTT $2 \mu \mathrm{l}$, RNAase $1 \mu \mathrm{l}$ ). Adding the reaction mixture to the RNA/primer mixture, mixing briefly, and then placed at room temperature for $2 \mathrm{~min}$. Adding $1 \mu \mathrm{L}$ (50 units) of SuperScript II RT to each tube, mix and incubate at $25^{\circ} \mathrm{C}$ for $10 \mathrm{~min}$. Incubate the tubes at $42^{\circ} \mathrm{C}$ for $50 \mathrm{~min}$, heat inactivate at $70^{\circ} \mathrm{C}$ for $15 \mathrm{~min}$, and were chilled on ice. Adding $1 \mu \mathrm{L}$ RNase $\mathrm{H}$ and incubated at $37^{\circ} \mathrm{C}$ for $20 \mathrm{~min}$. The reaction stored the $1 \mathrm{st}$ strand cDNA at $-20^{\circ} \mathrm{C}$ until use for real-time PCR. BUB1b (Forward): ACGTTATTAGAAAGAGCTGTAG, BUB1b (Reverse): CATATCCAAAGGCTCATTGC, ACTB (Forward): AGAGAAGTGGGGTGGCTTTT, ACTB (Reverse): GCCGAGGACTTTGATTGCAC. Data analysis was performed with the manufacturer-supplied software.

\section{Statistical analysis}

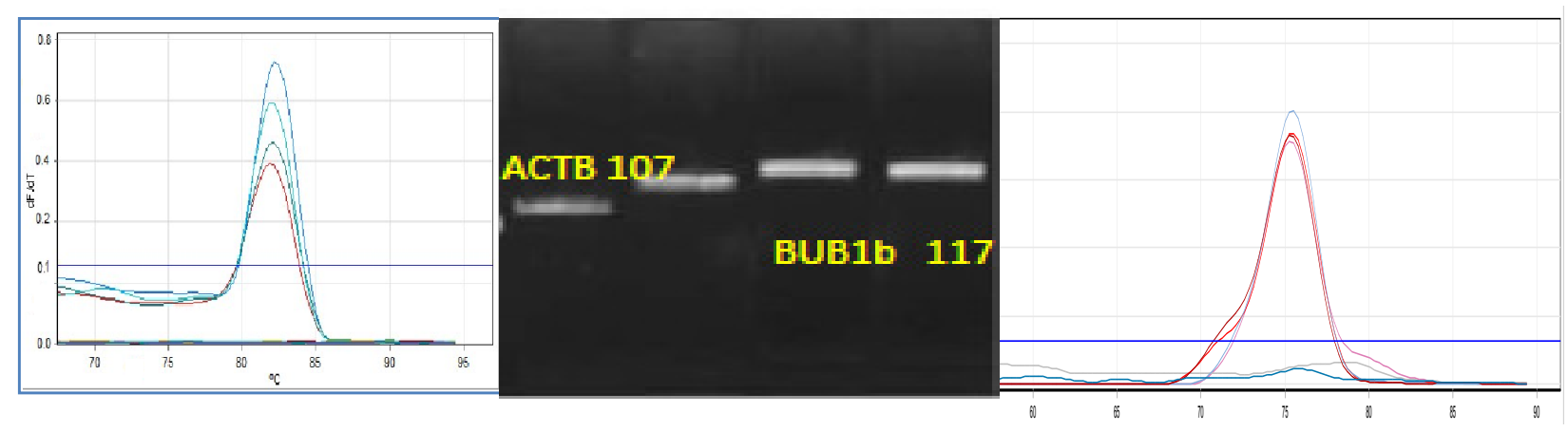

Figure 1 a

Figure 1 b

Figure 1 d

Figure 1a. The Melting Curve of RT-PCR Analysis for $\beta$-Actin Gene as Housekeeping Gene for Internal Control. Figure 1b. BUB1 Tm: $83^{\circ} \mathrm{C}$ Respectively.

Figure 1d. Determining the Specificity of RT-PCR Products; Electerophoresis of RT-PCR Products on 2\% Gel Agarose, for Beta-Actin, BUB1 Genes. 
Table 1. Comparison of Sentinel Lymph Node and Normal Breast Tissue for BUB1 Gene Expression

\begin{tabular}{rccccr}
\hline \multicolumn{5}{c}{ Exp. BUB1b } \\
\hline \multirow{3}{*}{ Valid } & & Frequency & Percent & Valid Percent & Cumulative Percent \\
& negative & 8.0 & 15.1 & 16.0 & 16.0 \\
& positive & 42.0 & 79.2 & 84.0 & 100.0 \\
\hline
\end{tabular}

Comparison of the results between various experimentally treated groups and their corresponding controls was carried out by SPSS .20 software with t-test and pearson chi-square. All comparisons were considered significant when $\mathrm{p}<0.05$.

\section{Results}

Sentinel lymph node samples were removed after surgery from breast of women with breast cancer. The average age of patients was 48.6 years. The results of pathology tests were negative in samples, which mean that the Sentinel lymph node samples were free of micro metastases. The second group of samples was obtained from healthy women without the disease, and the third group contains samples of metastasis. The ACTB gene was as the normalizing gene and its expression was examined in all groups along with test genes.

In current study, there was meaningful relation between expression of BUB1b with grade of tumor $(\mathrm{p}<0.01)$ and with stage $(\mathrm{p}=0.02)$, but not with ER status $(\mathrm{p}=0.19)$. Two group samples: 50 tumor micro metastasis and 50 adjacent normal breast cancer samples were obtained. Also 20 macro metastatic breast cancers as positive control and 50 samples from normal mastectomy breast tissue with no evidence for malignancy were included in this study. The beta actin gene was used as internal control for RT-PCR performance. As tumor pathological features, the stage of tumor is determined according to AJCC-02-TNM International criteria for classifying as $1=1,2=2 \mathrm{a}, 3=2 \mathrm{~b}, 4=3 \mathrm{a}, 5=3 \mathrm{~b}, 6=3 \mathrm{c}, 7=4$, where the current samples were in stage 1 and 2 and for grade of tumor, they are grouped as I,II,III according to pathological standards. The ER status, is reported as positive and negative. Moreover, the extent of expression of each gene, are evaluated with reduction or increase comparing to beta actin expression level (fold change). İn

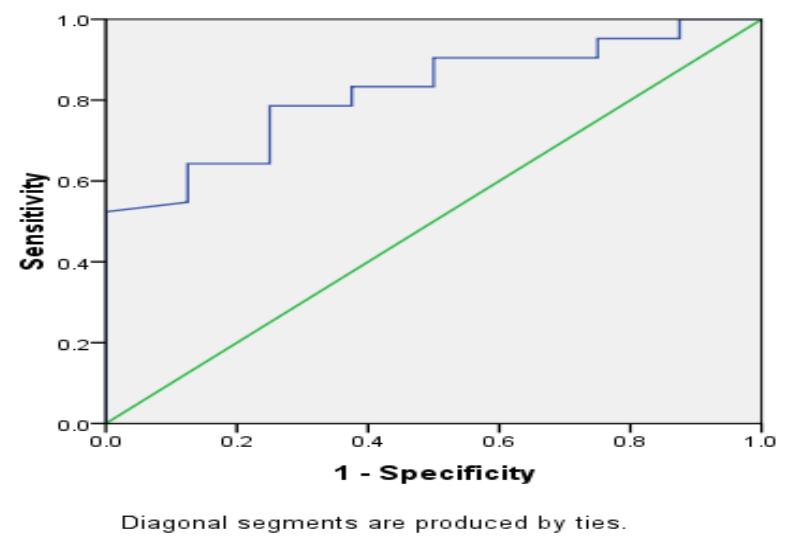

Figure 2. ROC Analysis for Test Accuracy for BUB1 Gene, Area Under Curve 0.82 evaluation of ER gene expression, about 58\% (29 sample) were ER negative and $42 \%$ (21 sample).

Real-time quantitative polymerase chain reaction results

The RT-PCR reaction was performed on all cancer and tumor samples as described earlier and the whole data were analysed statistically (Figures 1a, 1b, 1d).

In evaluation of tumor and normal samples for expression for BUB1 gene, $15.1 \%$ (8 sample) has not revealed expression and $79.2 \%$ (42 sample) has resulted expression $(\mathrm{p}<0.01)$ (Table1). In evaluation of BUB1 expression with ER status, there is no significant difference $(p=0.19)$, but there was a meaningful correlation between stage and BUB1 gene expression $(\mathrm{p}<0.01)$ and with tumor's grade, there was a significant difference too $(p=0.02)$. The results of ROC analysis for each gene is shown as follows in Figure 2.

\section{Discusion}

The cancer is the complex disease that has the variable pathological and cytological features (Bonora et al., 2015). The cytogenetic and molecular diagnosis is important challenge for prognosis and treatment of disease for physician (Ma et al., 2008). In the past study the application of molecular markers such HOXB13 made the prognosis much accurate that can predict the metastasis potential of cancer (Goetz et al., 2012). Wang and colleagues in 2005 reported the results of their studies, the use of gene expression profiling as a powerful tool to identify patients at risk of recurrence provided and accordingly can be better and less invasive treatment options should be taken. Regarding to their studies, microarray analysis in breast cancer samples, they found that 76 genes that the most important role plays in the progression of breast cancer and was highly informative in identifying patients who developed distant metastases within 5 years. The most strong correlation was found on 8 gene that founctionaly related with clinical features (Colak et al., 2013). For example in the work of Wang and coworkers (2009) on paraffin embedded breast cancer samples, it has shown the relation between mammaglobin gene and metastatic outcome of cancer. In another study, with RT-PCR on mama globin with blood sample and cancer tissue biopsy, the relation between low grades (I,II) with ER positive samples has revealed (Ouellette et al., 2004). Also, in BUB1 assessment, it was clear the inconstancy of this gene in colorectal at $14.5 \%$ of patients sample (Jaffrey et al., 2000). As the data shown in our study the evaluation of BUB1b, there was significant variation between normal and tumor samples $(p<0.01)$. Also the BUB1b expression is in relation with $\mathrm{Ki}-67$ marker and this protein is positive in $40 \%-50 \%$ of cancer 
cells (Ellis et al., 2001). The expression level of BUB1b is in correlation with distant metastasis and progression of breast carcinoma (Nishidate et al., 2004). the mechanism of BUB1b role in cancer is unclear but it may be due to its role in chromosomal segregation and it's reduced expression also may cause the chromosomal instability. Thus, BUB1b balanced expression is essential for cell homeostasis (Jeganathan et al., 2007). In addition, BUBb1 loss acts to inhibit the initiation of neoplastic growth in prostate epithelial cells, presumably by increasing apoptosis and reducing cell proliferation in early-stage neo plastic cells (Baker et al., 2009). This study shown that BUB $1 \mathrm{~b}$ expression assay can be used for micrometastasis molecular detection in some cancers (Pappa et al., 2014). In our study the BUB1b expression assay to group of patient with and without metastasis and directly compared together BUB1b expression showed over expression in 2 group but didn't show overexpression in normal breast and lymph nodes tissue.

In summary, the expression of BUB1b, gene was evaluated. Overexpression of BUB1b could be involved in invasion and genomic instability molecular pathways of cancer cells. The data from our studies on the detection of micro-metastasis in breast cancer patient without metastasis, with metastasis and normal lymph node tissue, detecting of BUB1b as molecular marker for early diagnosis. Clearly of BUB1b gene over expression in micro-metastasis and metastatic samples evidence and compared with normal breast and lymph nodes tissue and previous studies, suggest that changes in BUB1b gene expression can be used as a molecular marker to assess for accurate micro-metastasis and managing the treatment of breast cancer patients. However, to achieve more accurate results needs more data to draw firm conclusion in the field of cancer in an Iranian population (Movafagh et al., 2011; Varma et al., 1995; Movafagh et al., 2007).

\section{Acknowledgment}

The result of this research work is the thesis work of Neda Mansouri MSc Student. This work was supported by deputy research administration, Shahid Beheshti University of Medical Sciences, Tehran, Iran.” grant GR No: -451-20-11-1392.

\section{Conflict of interest}

The authors declare no conflict of interest.

\section{References}

Baker DJ, Jin F, Jeganathan KB, et al (2009). Whole chromosome instability caused by Bub1 insufficiency drives tumorigenesis through tumor suppressor gene loss of heterozygosity. Cancer Cell, 16, 475-86.

Bonora M, Wieckowsk MR, Chinopoulos C, et al (2015). Molecular mechanisms of cell death: central implication of ATP synthase in mitochondrial permeability transition. Oncogene, 34, 1608.

Colak D, Nofal A, AlBakheet A (2013). Age-specific gene expression signatures for breast tumors and cross-species conserved potential cancer progression markers in young women. culhane AC, ed. PLoS ONE, 8, e63204.
Daniele L, Annaratone L, Allia E, et al (2009). Technical limits of comparison of step-sectioning,immunohistochemistry and RT-PCR on breast cancer sentinel nodes: a study on methacarn-fixed tissue. J Cell Mol Med, 13, 4042-50.

Early Breast Cancer Trialists' Collaborative Group (EBCTCG) (2005). Effects of chemotherapy and hormonal therapy for early breast cancer on recurrence and15-year survival: an overview of the randomised trials. Lancet, 65, 1687-1717.

Ellis MJ, Coop A, Singh B, et al (2001). Letrozole is more effective neoadjuvant endocrine therapy than tamoxifen for ErbB-1-and/or ErbB-2-positive, estrogen receptor-positive primary breast cancer: evidence from a phase III randomized trial. J Clin Oncol, 19, 3808-16.

Fardmanesh H, Shekari M, Movafagh A, et al (2016). Upregulation of the double-stranded RNA binding protein DGCR8 in invasive ductal breast carcinoma. Gene, 58, 146-51.

Freedman RA, Kouri EM, West DW, et al (2015). Higher stage of disease is associated with bilateral mastectomy among patients with breast cancer: a population-based survey. Clin Breast Cancer, 16, 105-12.

Gerber B, Heintze K, Stubert J, et al (2011). Axillary lymph node dissection in early-stage invasive breast cancer: is it still standard today? Breast Cancer Res Treat, 128, 613-24.

Gillanders WE, Mikhitarian K, Hebert R, et al (2004). Molecular detection of micrometastatic breast cancer in histopathologynegative axillary lymph nodes correlates with traditional predictors of prognosis: an interim analysis of a prospective multi-institutional cohort study. Ann Surg J, 239, 828-37.

Goetz MP, Suman VJ, Ingle JN, et al (2006). A two-gene expression ratio of homeobox 13 and interleukin-17B receptor for prediction of recurrence and survival in women receiving adjuvant tamoxifen. Clin Cancer Res, 12, 2080-7.

Guillén-Paredes MP, Carrasco-González L, Cháves-Benito A, et al (2011). [One-step nucleic acid amplification (OSNA) assay for sentinel lymph node metastases as an alternative to conventional postoperative histology in breast cancer: A cost-benefit analysis]. Cir Esp, 89, 456-62.

Habel LA, Sakoda LC, Achacoso N, et al (2013). HOXB13:IL17BR and molecular grade index and risk of breast cancer death among patients with lymph nodenegative invasive disease. Breast Cancer Res, 15, R24.

Heidari MH, Porghasem M, Mirzaei N, et al (2014). The effect of high level natural ionizing radiation on expression of PSA, CA19-9 and CEA tumor markers in blood serum of inhabitants of Ramsar, Iran. J Environ Radioact, 128, 64-7.

Jaffrey RG, Pritchard SC, Clark C, et al (2000). Genomic instability at the BUB1 locus in colorectal cancer, but not in non-small cell lung cancer. Cancer Res, 60, 4349-52.

Ma XJ, Salunga R, Dahiya S, et al (2008). A five-gene molecular grade index and HOXB13:IL17BR are complementary prognostic factors in early stage breast cancer. Clin Cancer Res, 14, 2601-8.

Movafagh A, Maleki F, Fadaie S, et al (2007). Persistent unstable chromosomal aberration in lymphocytes of radiotherapy workers after 1 st mitotic division in Tehran, Iran. PJMS, 23, 254-60.

Movafagh A, Mirfakhraei R, Mousavi-Jarrahi A et al (2011). Frequent incidence of double minute chromosomes in cancers, with special up-to-date reference to leukemia. Asian Pac J Cancer Prev, 12, 3453-6.

Movafagh A, Hajifathali A, Isfahani F, et al (2012). Geographic heterogeneity of cytogenetic characteristics of acute myeloid leukemia in the early detection. Iran J Cancer Prev, 2, 85-9.

Movafagh A, Hajifathali A, Zamani M (2011). Secondary chromosomal bnormalities of de novo acute myeloid leukemia-a first report from the Middle East. Asian Pac J 
Cancer Prev, 12, 2991-4.

Nishidate T, Katagiri T, Lin ML, et al (2004). Genome-wide gene-expression profiles of breast-cancer cells purified with laser microbeam microdissection: identification of genes associated with progression and metastasis. Int J Oncol, 25, 797-819.

Ouellette RJ, Richard D, Maïcas E (2004). RT-PCR for mammaglobin genes, MGB1 and MGB2, identifies breast cancer micrometastases in sentinel lymph nodes. Am J Clin Pathol, 121, 637-43.

Pappa KI, Rodolakis A, Christodoulou I, et al (2014). Comparative assessment of lymph node micrometastasis in cervical, endometrial and vulvar cancer: insights on the real time qRT-PCR approach versus immunohistochemistry, employing dual molecular markers. Biomed Res Int, 187684.

Patani N, Mokbel K(2011). Clinical significance of sentinel lymph node isolated tumour cells in breast cancer. Breast Cancer Res Treat, 127, 325-34.

Shargh SA, Sakizli M, Khalaj V, et al (2014). Downregulation of E-cadherin expression in breast cancer by promoter hypermethylation and its relation with progression and prognosis of tumor. Med Oncol, 31, 250.

Seifi-Alan M, Shamsi R, Ghafouri-Fard S, et al (2014). Expression analysis of two cancer-testis genes, FBXO39 and TDRD4, in breast cancer tissues and cell lines. Asian Pac J Cancer Prev, 14, 6625-9.

Varma N, Varma S, Movafagh A, et al (1995). Unusual clonal cytogenetic abnormalities in aplastic anemia. Am J Hematol, 49, 256-7.

Wang Y, Klijn JG, Zhang Y, et al (2005). Gene-expression profiles to predict distant metastasis of lymph-node-negative primary breast cancer. Lancet, 365, 671-9.

Wang Z, Spaulding B, Sienko A, et al (2004). Mammaglobin, a valuable diagnostic marker for metastatic breast carcinoma. Int J Clin Exp Pathol, 2, 384-9. 